\title{
Charge density waves in cuprate superconductors beyond the
} critical doping

\author{
H. Miao $\mathbb{D}^{1 凶}$, G. Fabbris $\mathbb{D}^{2}$, R. J. Koch ${ }^{1}$, D. G. Mazzone $\mathbb{D i D}^{1,7}$, C. S. Nelson ${ }^{3}$, R. Acevedo-Esteves ${ }^{3}$, G. D. Gu $^{1}$, Y. Li ${ }^{1}$, T. Yilimaz $^{3}$, \\ K. Kaznatcheev ${ }^{3}$, E. Vescovo ${ }^{3}$, M. Oda ${ }^{4}$, T. Kurosawa ${ }^{4}{ }^{4}$, N. Momono ${ }^{5}$, T. Assefa ${ }^{1,8}$, I. K. Robinson (iD ${ }^{1,6}$, E. S. Bozin ${ }^{1}$, J. M. Tranquada (iD) \\ P. D. Johnson ${ }^{1}$ and M. P. M. Dean (iD) ${ }^{1 凶}$
}

\begin{abstract}
The unconventional normal-state properties of the cuprates are often discussed in terms of emergent electronic order that onsets below a putative critical doping of $x_{c} \approx 0.19$. Charge density wave (CDW) correlations represent one such order; however, experimental evidence for such order generally spans a limited range of doping that falls short of the critical value $x_{\mathrm{c}}$ leading to

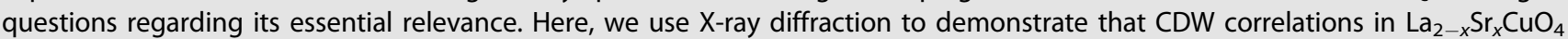
persist up to a doping of at least $x=0.21$. The correlations show strong changes through the superconducting transition, but no obvious discontinuity through $x_{c} \approx 0.19$, despite changes in Fermi surface topology and electronic transport at this doping. These results demonstrate the interaction between CDWs and superconductivity even in overdoped cuprates and prompt a reconsideration of the role of CDW correlations in the high-temperature cuprate phase diagram.
\end{abstract}

npj Quantum Materials (2021)6:31; https://doi.org/10.1038/s41535-021-00327-4

\section{INTRODUCTION}

The cuprate high- $T_{c}$ superconductors are often conceptualized as doped Mott insulators, in which the electronic ground state spontaneously breaks rotational and/or translational symmetry ${ }^{1-4}$. While cuprate CDW correlations were discovered over two decades $\mathrm{ago}^{5}$, their possible contribution to the material's anomalous electronic properties remains a matter of vigorous debate $^{1,6-10}$. This issue has gained increasing attention in light of the ubiquity of CDW order in different cuprate families ${ }^{5,11-19}$. The cuprate phase diagram, shown in Fig. 1a, shows that pseudogap, strange metal, and superconducting phases exist over an extensive doping range below a critical doping level of $x_{c} \approx 0.19$, above which the cuprate electronic properties become gradually more Fermi-liquid-like ${ }^{20-27}$. If CDW correlations are confined to underdoped cuprates, as previously suggested ${ }^{11-21}$, that would preclude the possibility of CDW correlations having an important role in the anomalous electronic properties. For instance, it has been argued that since CDW correlations disappear at $x \ll x_{C}$, the quantum critical point (QCP) at $x=x_{c}$ must be magnetic in nature $^{8}$. Tunneling spectroscopy studies have suggested a vestigial nematic QCP on a similar basis ${ }^{28,29}$. Very recent nuclear magnetic resonance results have reported the disappearance of spin glass behavior near $x_{c}{ }^{30}$. Whether this disappearance is associated with the loss of stripe correlations (i.e., coupled spin and charge density waves) remains unresolved. Moreover, the existence of CDW correlations is also crucial for the relevance of intertwined order. Many theoretical models for pair density wave superconducting states, for example, require the presence of CDW correlations ${ }^{1,31}$.

Studies of underdoped and optimally doped cuprates have shown that CDW correlations exist up to temperatures well above the nominal CDW transition temperature ${ }^{32,33}$. More recently, re- entrant charge order, disconnected from the CDW at lower doping, was observed in overdoped $(\mathrm{Bi}, \mathrm{Pb})_{2.12} \mathrm{Sr}_{1.88} \mathrm{CuO}_{6+\delta}{ }^{19}$. These results motivate a reconsideration of the cuprate phase diagram, in which CDW correlations may extend up to higher dopings than previously thought ${ }^{33}$. Herein, we address this issue by focusing on $\mathrm{La}_{2-x} \mathrm{Sr}_{x} \mathrm{CuO}_{4}$ (LSCOx) $(x=0.12,0.17,0.21$, and 0.25 ) single crystals in view of its particularly well-characterized transport properties and the feasibility of synthesizing high-quality samples across the entire phase diagram ${ }^{21,24-27}$ (see Methods section).

\section{RESULTS \\ Electronic structure}

Previous measurements of LSCO established the presence of a critical doping $x_{\mathrm{c}} \sim 0.19$, which was defined as the doping above which the electronic transport acquires partial Fermi-liquid-like properties ${ }^{21,24}$. This point coincides with, and is perhaps related to, the doping where the Fermi surface (FS) topology undergoes a Lifshitz transition ${ }^{34,35}$. To prove the Sr doping, $x$, is consistent with previous studies, and that we indeed access the $x>x_{c}$ region of the overdoped phase diagram, we show the electronic structure evolution with doping in Fig. 1b-e. The angle-resolved photoemission spectroscopy (ARPES) methods used are described in the Methods section. Figure $1 \mathrm{~b}$ illustrating the two-dimensional electronic structure. At low doping, to the extent that a FS exists, it is hole-like and centered at the Brillouin zone corner. With increasing hole concentration, the chemical potential drops and eventually passes through the saddle point. This results in a Lifshitz transition to an electron-like FS at the Brillouin zone center. In LSCO, the saddle point is three-dimensional with small $k_{z}$ dispersion. Near $x_{\mathrm{C}}$ the saddle point coincidentally crosses the

\footnotetext{
${ }^{1}$ Condensed Matter Physics and Materials Science Department, Brookhaven National Laboratory, Upton, NY 11973 , USA. ${ }^{2}$ Advanced Photon Source, Argonne National Laboratory, Argonne, IL 60439, USA. ${ }^{3}$ National Synchrotron Light Source II, Brookhaven National Laboratory, Upton, NY 11973, USA. ${ }^{4}$ Department of Physics, Hokkaido University, Sapporo 060-0810, Japan. ${ }^{5}$ Department of Sciences and Informatics, Muroran Institute of Technology, Muroran 050-8585, Japan. ${ }^{6}$ London Centre for Nanotechnology, University College, Gower St., London WC1E 6BT, UK. 'Present address: Laboratory for Neutron Scattering and Imaging, Paul Scherrer Institute, 5232 Villigen, Switzerland. ${ }^{8}$ Present address: Stanford Institute for Materials and Energy Sciences, SLAC National Accelerator Laboratory, Menlo Park, CA 94025, USA. ${ }^{凶}$ email: hmiao@bnl.gov; mdean@bnl.gov
} 

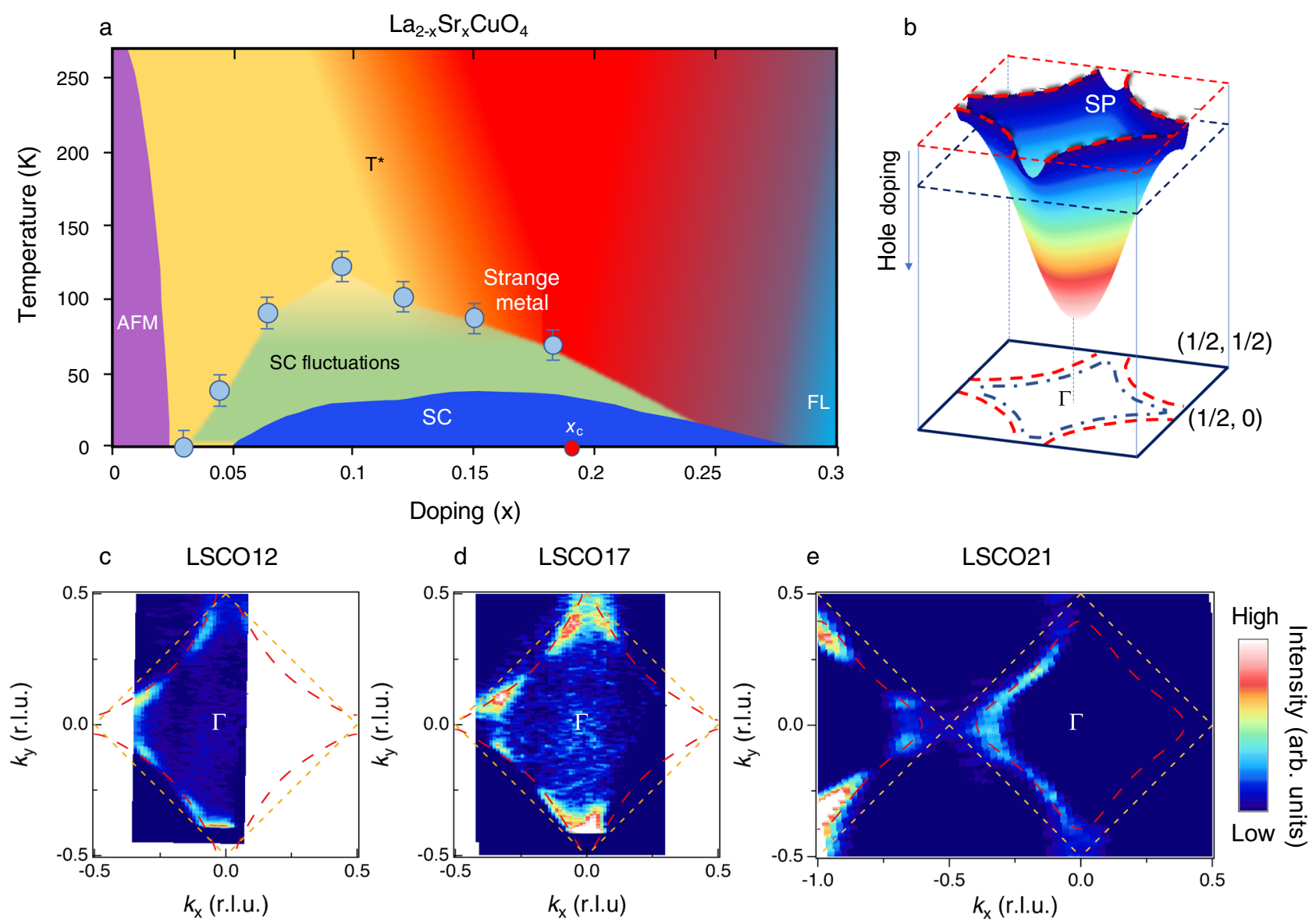

Fig. 1 Doping-dependent electronic structure of LSCO. a Phase diagram of the hole-doped cuprates, constructed from magnetization, Nernst effect, and resistivity data for LSCO ${ }^{20,52} \cdot T^{*}$ is the extracted pseudogap onset temperature ${ }^{20,52}$. b Schematic band structure of LSCO. The Fermi energy, $E_{F}$, crosses the anti-nodal saddle point (labeled as SP) near $x_{c} \approx 0.19$ triggering a Lifshitz transition. c-e Fermi surface topology of LSCO12, LSCO17, and LSCO21. The intensity plots are obtained by integrating the spectra within \pm 10 meV of $E_{F}$. Orange dashes outline the antiferromagnetic Brillouin zone. Red dashed contours represent a tight-binding fit of the Fermi surface (see Supplementary note 1). The data shown in c-e were collected at $11 \mathrm{~K}$.

Fermi level. It should be noted that the carrier concentration determined by the FS area is significantly larger than the nominal $\mathrm{Sr}$ doping. The origin of this effect remains unresolved ${ }^{34,35}$. Nevertheless, the well-established FS evolution in LSCO can be used to confirm the $\mathrm{Sr}$ doping in this study. Figure 1c-e shows ARPES measurements for LSCO12, LSCO17, and LSCO21. An electron-like FS is observed in LSCO21, consistent with $x_{\mathrm{C}}=0.19$ and in agreement with previous ARPES studies ${ }^{34,35}$.

\section{CDW order}

Having confirmed the electronic structure, we now present our main experimental finding of CDW correlations beyond $x_{\mathrm{c}}$. Figure 2 plots X-ray reciprocal space scans for LSCO21 at $T=16 \mathrm{~K}$, where reciprocal space is defined in terms of scattering vector $Q=(H, K$, $L$ ) using effective tetragonal lattice constants $a=b \approx 3.8 \AA$ and $c \approx$ 13.2 A. High sensitivity is achieved by exploiting the high brightness of the National Synchrotron Light Source II (NSLS-II) and by careful configuration of the detection system to suppress background signal (see Methods section). Superlattice peaks are observed at $(0.235,0,12.5)$, and equivalent locations, along both the $H$ and $K$ directions [Fig. 2a, b]. The observed $H=0.235$ matches the CDW wavevector in underdoped LSCO $^{16-18,36}$ and is consistent with the charge stripe picture $^{5}$. The peaks are symmetric with respect to $\pm H$ and $K$ and are observed in multiple Brillouin zones including $( \pm 0.235,0, L)$ for $L=8.5$ and 12.5. An $L-$ scan along $Q=(-0.235,0, L)$ [Fig. 2d] reveals that the CDW intensity is broadly peaked at half-integer $L$ similar to underdoped $\mathrm{LSCO}^{17,18,37}$. These results demonstrate the presence of CDW correlations beyond $x_{c}$. Subsequent inelastic X-ray scattering studies show that the CDW is associated with phonon softening even in the overdoped regime ${ }^{38}$.

\section{CDW temperature dependence}

Figure 3 summarizes the doping and temperature dependence of the CDW correlations. In Fig. 3a, Lorentzian-squared fits to the data are shown, which are parameterized in terms of amplitude, $I_{\text {CDW }}(T)$, and in-plane correlation length, $\xi_{\|}(T)=1 / \mathrm{HWHM}$ (where HWHM is half-width at half-maximum) (see Supplementary note 2). Since domain formation can lead to transverse peak splitting in LSCO [c.f. refs. ${ }^{16,18,36}$ and Fig. 3a], we scanned through the peaks in all three reciprocal space directions. Two Lorentzian-squared functions displaced in the $K$ (transverse) direction were used, where necessary, to account for the full intensity distribution. Peak widths and correlation lengths are determined using the $H$ (longitudinal) cut. $I_{\mathrm{CDW}}(T)$ is found to be largest near $T_{\mathrm{SC}}$ for all dopings [Fig. 3b]. Above $T_{\mathrm{SC}}$, both $I_{\mathrm{CDW}}$ and $\xi_{\|}(T)$ decrease with increasing temperature but remain finite up to at least $T=90 \mathrm{~K}$ [Fig. 3a]. In agreement with previous X-ray diffraction studies of $\operatorname{LSCO}^{16,17,36}$, the correlation length can be separated into a marginally-ordered regime where $\xi_{\|}(T)$ is $\sim 4$-unit cells (about one period of the CDW order), and a strongly $T$-dependent regime where $\xi_{\|}(T)$ continues to expand until superconductivity 


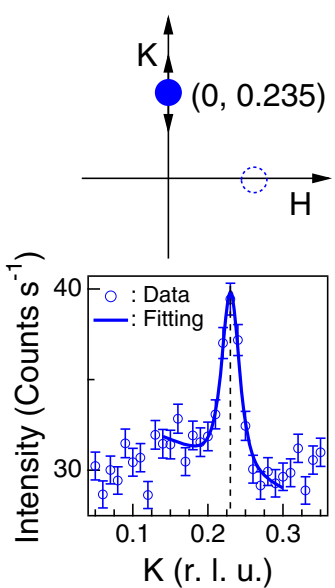

b

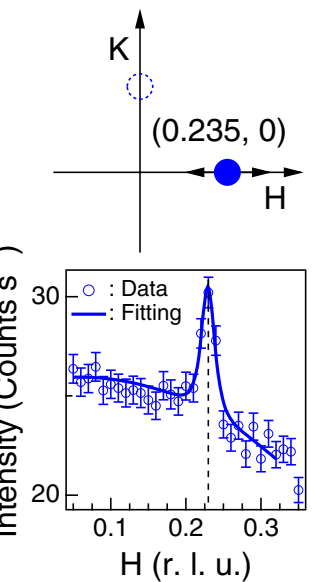

C

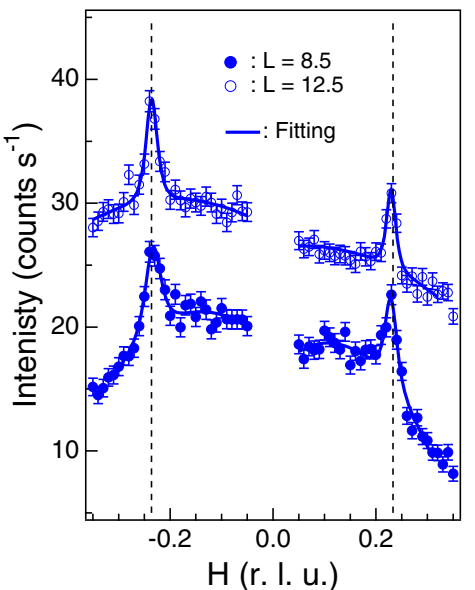

d

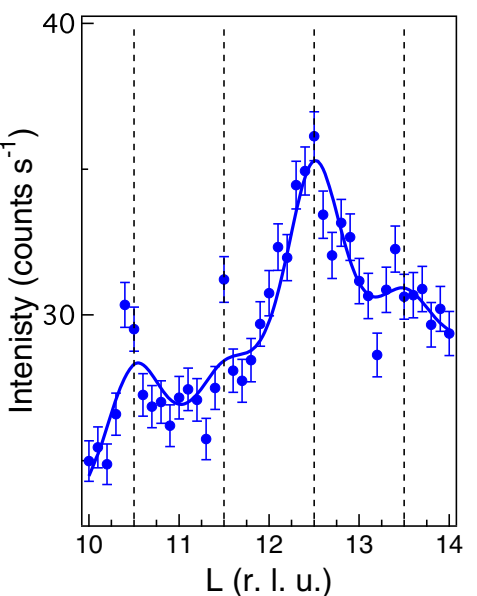

Fig. 2 Discovery of a CDW beyond $\boldsymbol{x}_{\mathbf{c}}$. a, b X-ray diffraction measurements of LSCO21 at $T=16 \mathrm{~K}$ along $(0, K, 12.5)$ and $(H, 0,12.5)$. Supperlattice peaks are observed at $(0,0.235,12.5)$ and $(0.235,0,12.5)$. The $H$-scans in c reveal further CDW peaks at $( \pm 0.235,0, L)$ for $L=8.5$ and 12.5. The data at $L=8.5$ are offset by -10 counts $\mathrm{s}^{-1}$ for visibility. $\mathbf{d}$ The $L$-dependence of the intensity along $(-0.235,0, L)$ demonstrates poorly correlated out-of-phase CDW stacking along the $c$-axis. Solid lines are fits to the experimental data as described in the text and Supplementary note 2. Error bars are one standard deviation based on Poissonian statistics.
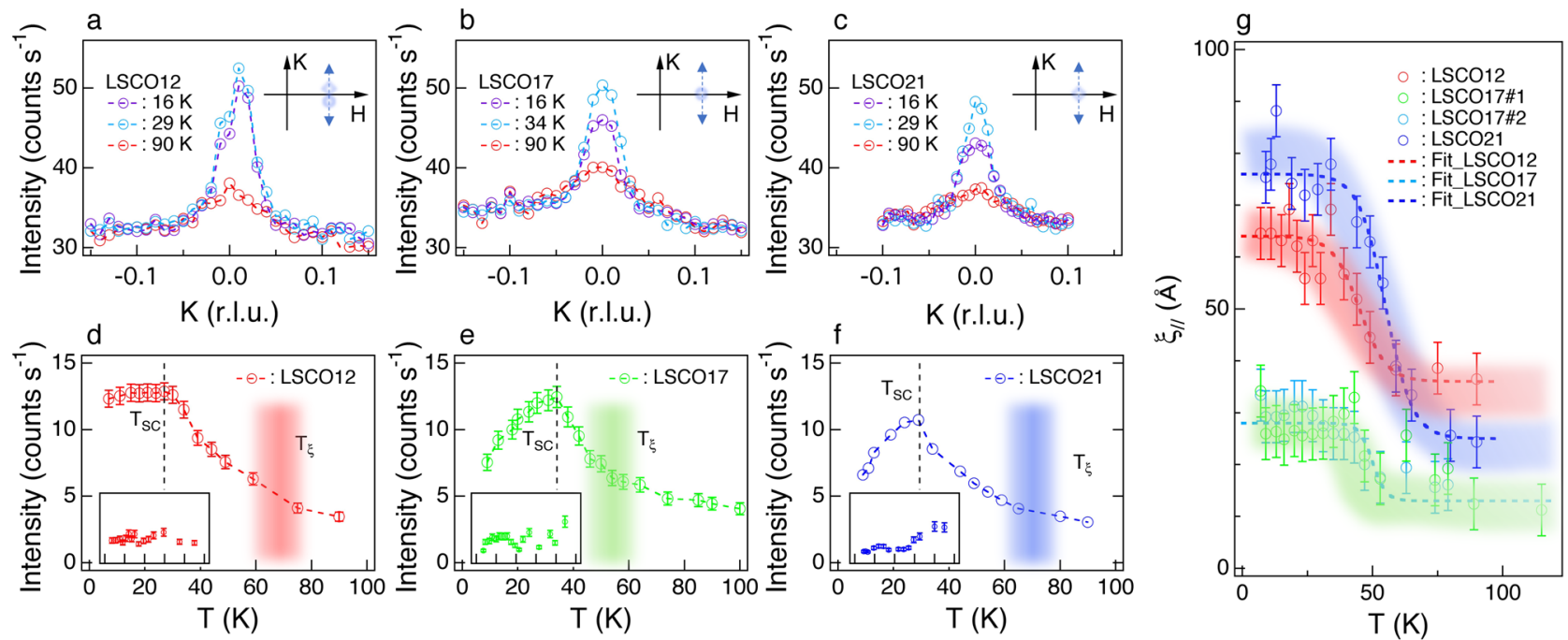

Fig. 3 CDW temperature dependence. Doping dependence of the CDW peak intensity for temperatures $T<T_{\mathrm{SC}}, T \approx T_{\mathrm{SC}}$, and $T>T_{\mathrm{SC}}$ for a LSCO12, b LSCO17, and $\mathbf{c}$ LSCO21. The inset of each panel represents the respective cut in reciprocal space. All data were taken at $L=8.5$. Temperature dependence of the CDW intensity in LSCO for $\mathbf{d}$ LSCO12, e LSCO17, and $\mathbf{f} L S C O 21$. The shaded area corresponds to $T_{\xi}$ where the in-plane CDW correlation length, $\xi_{\|}$, starts to increase [as determined in $\mathbf{g}$ ]. The main panels show peak height intensity and the insets show integrated intensity. $\mathbf{g}$ Temperature dependence of $\xi_{\|}$. The colored shaded curves are phenomenological fittings, $a+\frac{b}{1+e^{\left(T-T_{0}\right) / 4 \Delta T}}$, of the temperature-dependent $\xi_{\|}$for different dopings. Here we define $T_{\xi}=T_{0}+\Delta T$. $\xi_{\|}$increases with decreasing temperature for $T_{\mathrm{SC}}<T<T_{\varepsilon}$. Two independent measurements of LSCO17 samples at different beamlines show consistent suppression of $\xi_{\|}$and $T_{\xi}$, indicating that systematic errors are minimal. Due to the short correlation length, the uncertainty of $T_{\xi}$ may be larger in LSCO17 than other dopings. Error bars are one standard deviation from either Poissonian statistics or least-squares fitting.

intervenes. We refer to the CDW in the $T$-independent regime as "precursor" CDW correlations in the sense that they come before the emergence of a stronger, more correlated CDW at low temperatures. Note that for these measurements we do not have the energy resolution to directly distinguish between dynamic and static correlations. The short correlation length and quasitemperature-independent nature of the percursor CDW indicates that it might be dynamic in nature. This phenomenology is consistent with resonant inelastic X-ray scattering (RIXS) scattering studies of $\mathrm{La}_{2-x} \mathrm{Ba}_{x} \mathrm{CuO} 4$ (LBCOx) and $\mathrm{YBa}_{2} \mathrm{Cu}_{3} \mathrm{O}_{6+\delta}$ which show a similar two-stage CDW formation ${ }^{32,33,39,40}$. While the CDW evolves smoothly from LSCO12 to LSCO21, both $\xi_{\|}$and the onset temperature of the longer-range $\mathrm{CDW}, T_{\varepsilon}$, are suppressed in the overdoped regime around $x_{c}$ [Figs. $3 c$ and 4]. The $Q$-integrated scattering intensity, as estimated by $l_{\mathrm{CDW}} \xi_{\|}^{2}$, shows minimal variation through $T_{\mathrm{SC}}$, indicating that while superconductivity alters the CDW correlation length, it does not strongly suppress the order parameter. We do not observe any CDW correlations in our high-sensitivity $X$-ray measurements at $x=0.25$ (see Supplementary note 3 ).

Previous measurements of the same $x=0.12$ sample allow us to compare the CDW order parameter, taken to be captured by the total $Q$-integrated scattering intensity, to other cuprate systems ${ }^{16}$. The CDW order parameter of LSCO12 is only four times weaker than 


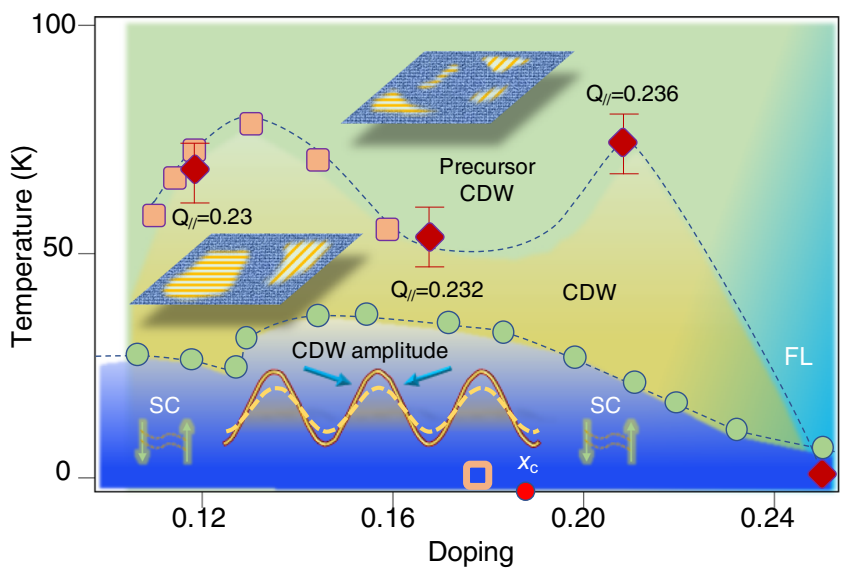

Fig. 4 Illustration of the extent of CDW correlations in the cuprate phase diagram suggested by this work. Green-yellow tones represent our main result-the presence of CDW correlations, from $0.12<x<0.21$. Green denotes the precursor CDW, which appear at high temperature and which have a correlation length of approximately one CDW period $32,33,39,40$. At lower temperature, the correlations start to grow into larger CDW domains, as evidenced by the increased correlation length, which we denote by the yellow tone. Red points mark where the correlation length starts to increase. This should be considered an approximate cross-over and not over-interpreted as a well-defined phase transition. At lower temperatures still, bulk $d$-wave superconductivity intervenes at $T_{\mathrm{sC}}$ whereupon both the CDW amplitude and the correlation length saturate or start to decrease. The doping dependence reveals an anticorrelation between $T_{\xi}$ and $T_{\mathrm{SC}}$, providing evidence for an interaction between the CDW and superconductivity. This is illustrated by the cartoon in the bottom of the diagram in which superconducting pairing (green spin pairs) suppress the CDW (yellow solid and dashed sinusoidal curves). The CDW intensity disappears in heavily overdoped LSCO25, where a Fermi-liquid-like state is recovered (Supplementary notes 3 and 5). The red diamonds reflect the present study. Pink squares and green circles are data from previous work ${ }^{16,17,36,52}$.

$\mathrm{La}_{1.875} \mathrm{Ba}_{0.125} \mathrm{CuO}_{4}$ (which has the strongest zero-field CDW order). With increasing doping, the LSCO CDW becomes somewhat stronger for $x=0.17$ and drops appreciably for $x=0.21$ (see Supplementary note 4). Consequently, CDW correlations can have an appreciable effect on the physics of LSCOx for dopings through $x_{c}$.

\section{DISCUSSION}

Figure 4 summarizes our main observations-that CDW correlations exist far into the overdoped regime of the cuprate phase diagram. This immediately yields three important consequences for LSCO. Firstly, very similar CDW properties are observed either side of the Lifshitz transition. This provides a vivid demonstration that CDW correlations cannot be explained within a weak coupling FS nesting picture nor Friedel oscillations. Instead, the nearly constant $Q_{\mathrm{CDW}}$ for dopings $x \geq 0.125$ support strong coupling mechanisms, which date back to seminal work in the late $1980 \mathrm{~s}^{2-4}$. In these mechanisms, one considers the balance between Coulomb interactions and kinetic energy. When doping a Mott insulator, holes can save energy by clustering together as this breaks fewer magnetic bonds than widely dispersed holes. At the same time, this clustering is disfavored by the increased Coulomb repulsion and kinetic energy reduction. Since these different interactions act on different lengthscales, the overall minimum energy solution is expected to involve a spatially modulated state. Modern numerical solutions of the Hubbard model further support this idea ${ }^{41-43}$ and models based on filled stripes can reproduce a doping-independent CDW wavevector from $x=1 / 8$ to $x=1 / 4^{44}$. We also note that precursor CDW correlations are emerging as a ubiquitous feature for many cuprates, including LSCO in this study, underdoped $\mathrm{LBCO}^{40}$, underdoped and optimally doped $\mathrm{YBCO}^{33}$, underdoped $\mathrm{Bi} 2212^{45^{\prime}}$, and $\mathrm{HgBa}_{2} \mathrm{CuO}_{4+\delta} 46$. In underdoped and optimally doped $\mathrm{YBCO}$, the precursor correlations appear to exist at the same wavevector around 0.3 r.l.u. different to the doping-dependent low-temperature $\mathrm{CDW}^{33}$. It would consequently be interesting to consider a possible role for strong coupling mechanisms for all cuprates. An obviously desirable experiment would be to test whether other cuprates, such as YBCO, also exhibit CDW correlations up to similarly high dopings as LSCO. Such experiments are, however, currently held back by challenges in stabilizing high-quality heavily overdoped YBCO crystals. The robust presence of CDW correlations in LSCO seen here as a function of temperature and doping, as well as the fact that model Hamiltonian calculations reliably predict CDW correlations ${ }^{41-43}$, would point towards their likely presence. The issue of differing wavevectors in different cuprates would, however, not necessarily be solved by such an experiment. In this regard, it is important to point out the lowtemperature ordering wavevector can be influenced by coupling between the CDW and spin correlations or coupling between the CDW and the lattice, as has been suggested theoretically ${ }^{47}$, so differences in CDW wavevectors could arise from secondary interactions rather than necessarily indicating a distinct origin for the correlations. Prior work has pointed towards this as a possible explanation for temperature-induced changes in CDW wavevector in $\mathrm{LBCO}^{32}$.

A second immediate conclusion is that the continuous evolution of the CDW correlations is inconsistent with the proposed QCP that is associated with $x_{c}$ arising from CDW or coupled CDW/spin density wave order ${ }^{6,10}$. Such theories can still be excluded even if one postulates a very narrow range of criticality around $x_{C}$, since they require either a disappearance or a symmetry change of the CDW through $x_{\mathrm{c}}$.

Last but not least, the disappearance of CDW in LSCO25 suggests that the CDW dome in LSCO terminates between $x=0.21$ and 0.25 , where the Fermi-liquid behavior starts to recover ${ }^{21,24,25}$. This is, again, consistent with a strong coupling CDW mechanism as Coulomb repulsion is largely screened in the Fermi-liquid state. We note that in LSCO, the structural hightemperature tetragonal to low-temperature orthorhombic (LTO) phase transition also terminates near $x=0.21^{48}$. It has been argued that the local LTO distortion may help to stabilize the $\mathrm{CDW}^{49}$. The persistence of CDW correlations up to $x=0.21$ observed in this study is consistent with this scenario and indicates that electron-phonon coupling might be an important ingredient for the CDW formation ${ }^{38,39}$.

The observations herein also urge a re-examination of the potential role of CDWs in the anomalous electronic properties of the cuprates. CDW correlations are a prerequisite (but not a proof) of several prominent theories of cuprate properties, which would be expected to apply across the phase diagram and not just in the underdoped region where CDW correlations have been studied extensively in the past. This include the possibility that CDW correlations play a key role in the electronic transport properties $^{6,10}$. Theories of pair density wave order ${ }^{1,31,50,51}$, which predict competition between the CDW and uniform $d$-wave superconductivity, also fall into this category. As shown in Fig. 3, neither the CDW peak intensity nor the CDW correlation length shows the type of divergent-behavior associated with a typical phase transition. This behavior is consistent with a possible fluctuating CDW component, potentially influencing cuprate transport properties ${ }^{24-27,33}$.

Finally, we note that a charge Bragg peak has recently been observed in overdoped $(\mathrm{Bi}, \mathrm{Pb})_{2.12} \mathrm{Sr}_{1.88} \mathrm{CuO}_{6+\delta}(\mathrm{Bi} 2201)$, with a maximum doping comparable to that observed here ${ }^{19}$. This state, termed re-entrant charge order, has several properties that are different to CDW states in LSCO and other cuprates. Re-entrant 
charge order appears to exist only in an isolated region of the overdoped phase diagram, disconnected from the underdoped CDW order. The correlation length and temperature scale of this state are also far higher than other cuprates. Intriguingly, no interaction between re-entrant charge order and superconductivity is observed in Bi2201. In contrast, similarly well-correlated CDW states are associated with a strong suppression of superconductivity. All these behaviors are in strong contrast with the CDW in overdoped LSCO, where the CDW wavevectors, correlation length, and temperature dependence evolve smoothly from the properties of underdoped LSCO and strongly intertwine with superconductivity and low-temperature transport. Based on the electronic structure of $\mathrm{Bi} 2201$ and the wavevector of re-entrant charge order around 0.1 r.l.u., which extrapolates roughly linearly from the underdoped CDW wavevector, re-entrant charge order was proposed to arise from a van Hove singularity ${ }^{19}$. The overdoped CDW in LSCO appears to have no connection to this mechanism, since the CDW remains unchanged regardless of the proximity to the van Hove singularity at $x=x_{c}$. Instead, our observations support strong coupling mechanisms.

In summary, high-sensitivity X-ray measurements have revealed that cuprate CDW correlations persist across almost the whole cuprate doping phase diagram, despite dramatic changes in the transport properties and FS topology, before disappearing when Fermi-liquid-like properties are restored. We have shown that these correlations impact superconductivity even in overdoped cuprates, suggesting that CDW correlations can have a far more extensive role in the cuprate phase diagram than previously envisaged, prompting investigations of CDW correlations in other overdoped cuprates. The discovery of CDWs beyond $x_{c}$ is confirmed by subsequent RIXS studies, which uncovered an unusual coupling between the CDW and lattice vibrations ${ }^{38}$.

\section{METHODS}

\section{Samples}

Single crystals of $\mathrm{La}_{2-x} \mathrm{Sr}_{x} \mathrm{CuO}_{4}(x=0.12,0.17,0.21$, and 0.25) were grown by the traveling-solvent floating-zone method. For each composition, a single feed rod of $20-25 \mathrm{~cm}$ long was used, the first few centimeters of which was removed and discarded after growth. The remaining rod was annealed in flowing $\mathrm{O}_{2}$ at $980^{\circ} \mathrm{C}$ for 1 week. The superconducting transition temperatures, $28,37,30$, and $10 \mathrm{~K}$ were determined by $\mathrm{dc}$ magnetization measurements in an applied field of $1 \mathrm{mT}$ (after cooling in zero field). Our tight-binding fits to our ARPES measurements of these samples, described in Supplementary note 1, confirm the hole concentration matches the strontium content $x$.

\section{ARPES}

ARPES measurements were performed at the 21-ID-1 beamline of the NSLS-II using a Scienta-DA30 analyzer. Due to the small incident beam spot-size (less than $10 \times 10 \mu \mathrm{m}^{2}$ ), both the sample position and the incident light angle are fixed during the measurement. The ARPES intensity maps are obtained using the mapping-mode of the DA30 analyzer, which can cover $30^{\circ}$ of cone acceptance without sample rotation. All samples were cleaved in-situ and measured at $11 \mathrm{~K}$ within a vacuum better than $7 \times 10^{-11} \mathrm{mbar}$. The photon energy was set to $60 \mathrm{eV}$ for LSCO12 and LSCO17 with $18 \mathrm{meV}$ energy resolution. To confirm the FS of LSCO21 is a closed loop at the $\Gamma$ point, we set the photon energy to $195 \mathrm{eV}$ for LSCO21 with $90 \mathrm{meV}$ energy resolution. At this energy, we were able to cover the second Brillouin zone without sample rotation. The chemical potential is calibrated based on the ARPES spectra on Silver that are recorded before and after the ARPES measurement.

\section{Non-resonant hard X-ray scattering}

High-precision X-ray scattering measurements were performed at the insitu and resonant 4-ID beamline of NSLS-II and 4-ID-D beamline of the Advanced Photon Source (APS). The incident photon energy was set to $8.98 \mathrm{keV}$; slightly below the $\mathrm{Cu} K$-edge to minimize the fluorescence background. The measurements at NSLS-II were carried out with an avalanche photodiode detector. A LiF(004) crystal analyzer was used to

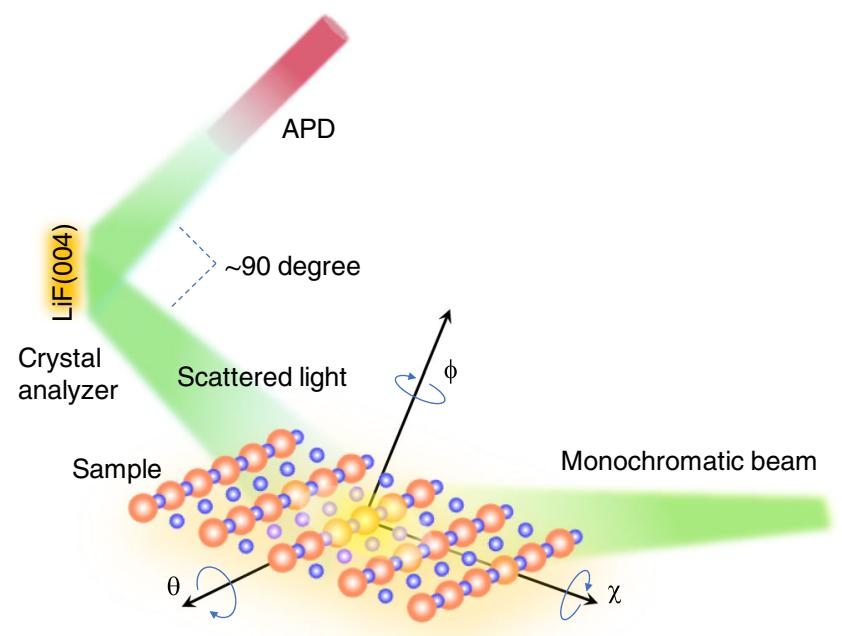

Fig. 5 Experimental setup. Illustration of the experimental geometry at the 4-ID beamline of NSLS-II.

further suppress the background signal (Fig. 5). The measurements at the APS used a Vortex Si drift detector without any crystal analyzer.

\section{DATA AVAILABILITY}

Data are available from the corresponding author upon reasonable request.

Received: 16 October 2020; Accepted: 23 February 2021; Published online: 19 March 2021

\section{REFERENCES}

1. Fradkin, E., Kivelson, S. A. \& Tranquada, J. M. Colloquium: theory of intertwined orders in high temperature superconductors. Rev. Mod. Phys. 87, 457-482 (2015).

2. Zaanen, J. \& Gunnarsson, O. Charged magnetic domain lines and the magnetism of high- $T_{c}$ oxides. Phys. Rev. B 40, 7391-7394 (1989).

3. Machida, K. Magnetism in $\mathrm{La}_{2} \mathrm{CuO}_{4}$ based compounds. Physica C Supercond. 158 192-196 (1989).

4. Emery, V. J., Kivelson, S. A. \& Lin, H. Q. Phase separation in the $t-J$ model. Phys. Rev. Lett. 64, 475-478 (1990).

5. Tranquada, J., Sternlieb, B., Axe, J., Nakamura, Y. \& Uchida, S. Evidence for stripe correlations of spins and holes in copper oxide superconductors. Nature 375, 561-563 (1995).

6. Castellani, C., Di Castro, C. \& Grilli, M. Singular quasiparticle scattering in the proximity of charge instabilities. Phys. Rev. Lett. 75, 4650-4653 (1995).

7. Sachdev, $\mathrm{S}$. Where is the quantum critical point in the cuprate superconductors? Phys. status solidi B 247, 537-543 (2010).

8. Doiron-Leyraud, N. \& Taillefer, L. Quantum critical point for stripe order: an organizing principle of cuprate superconductivity. Physica C Supercond. 481, 161-167 (2012).

9. Sebastian, S. E. \& Proust, C. Quantum oscillations in hole-doped cuprates. Annu. Rev. Condens. Matter Phys. 6, 411-430 (2015).

10. Caprara, S., Di Castro, C., Seibold, G. \& Grilli, M. Dynamical charge density waves rule the phase diagram of cuprates. Phys. Rev. B 95, 224511 (2017).

11. Hoffman, J. et al. A four unit cell periodic pattern of quasi-particle states surrounding vortex cores in $\mathrm{Bi}_{2} \mathrm{Sr}_{2} \mathrm{CaCu}_{2} \mathrm{O}_{8+\delta}$. Science 295, 466-469 (2002).

12. Howald, C., Eisaki, H., Kaneko, N. \& Kapitulnik, A. Coexistence of periodic modulation of quasiparticle states and superconductivity in $\mathrm{Bi}_{2} \mathrm{Sr}_{2} \mathrm{CaCu}_{2} \mathrm{O}_{8+\delta}$. Proc. Natl. Acad. Sci. USA 100, 9705-9709 (2003).

13. Ghiringhelli, $\mathrm{G}$. et al. Long-range incommensurate charge fluctuations in $(\mathrm{Y}, \mathrm{Nd})$ $\mathrm{Ba}_{2} \mathrm{Cu}_{3} \mathrm{O}_{6+x}$. Science 337, 821-825 (2012).

14. Comin, R. et al. Charge order driven by Fermi-arc instability in $\mathrm{Bi}_{2} \mathrm{Sr}_{2-x} \mathrm{La}_{x} \mathrm{CuO}_{6+\delta}$ Science 343, 390-392 (2014).

15. Tabis, W. et al. Charge order and its connection with Fermi-liquid charge transport in a pristine high- $T_{c}$ cuprate. Nat. Commun. 5, 5875 (2014). 
16. Thampy, V. et al. Rotated stripe order and its competition with superconductivity in $\mathrm{La}_{1.88} \mathrm{Sr}_{0.12} \mathrm{CuO}_{4}$. Phys. Rev. B 90, 100510 (2014).

17. Croft, T. P., Lester, C., Senn, M. S., Bombardi, A. \& Hayden, S. M. Charge density wave fluctuations in $\mathrm{La}_{2-x} \mathrm{Sr}_{x} \mathrm{CuO}_{4}$ and their competition with superconductivity. Phys. Rev. B 89, 224513 (2014).

18. Christensen, N. B. et al. Bulk charge stripe order competing with superconductivity in $\mathrm{La}_{2-x} \mathrm{Sr}_{x} \mathrm{CuO}_{4} \quad(x=0.12)$ Preprint at https://arxiv.org/abs/ 1404.3192 (2014).

19. Peng, $\mathrm{Y}$. et al. Re-entrant charge order in overdoped $(\mathrm{Bi}, \mathrm{Pb})_{2.12} \mathrm{Sr}_{1.88} \mathrm{CuO}_{6+\delta}$ outside the pseudogap regime. Nat. Mater. 17, 697-702 (2018).

20. Wang, Y., Li, L. \& Ong, N. P. Nernst effect in high- $T_{\mathrm{c}}$ superconductors. Phys. Rev. $B$ 73, 024510 (2006).

21. Cooper, R. A. et al. Anomalous criticality in the electrical resistivity of $\mathrm{La}_{2-x} \mathrm{Sr}_{x} \mathrm{CuO}_{4}$. Science 323, 603-607 (2009).

22. Keimer, B., Kivelson, S., Norman, M., Uchida, S. \& Zaanen, J. From quantum matter to high-temperature superconductivity in copper oxides. Nature 518, 179-186 (2015).

23. Ramshaw, B. J. et al. Quasiparticle mass enhancement approaching optimal doping in a high- $T_{c}$ superconductor. Science 348, 317-320 (2015).

24. Badoux, S. et al. Critical doping for the onset of Fermi-surface reconstruction by charge-density-wave order in the cuprate superconductor $\mathrm{La}_{2-x} \mathrm{Sr}_{x} \mathrm{CuO}_{4}$. Phys. Rev. X 6, 021004 (2016).

25. Giraldo-Gallo, P. et al. Scale-invariant magnetoresistance in a cuprate superconductor. Science 361, 479-481 (2018).

26. Boebinger, G. S. et al. Insulator-to-metal crossover in the normal state of $\mathrm{La}_{2-x} \mathrm{Sr}_{x} \mathrm{CuO}_{4}$ near optimum doping. Phys. Rev. Lett. 77, 5417-5420 (1996).

27. Michon, B. et al. Thermodynamic signatures of quantum criticality in cuprate superconductors. Nature 567, 218-222 (2019).

28. Nie, L., Tarjus, G. \& Kivelson, S. A. Quenched disorder and vestigial nematicity in the pseudogap regime of the cuprates. Proc. Natl. Acad. Sci. USA 111, 7980-7985 (2014).

29. Mukhopadhyay, S. et al. Evidence for a vestigial nematic state in the cuprate pseudogap phase. Proc. Natl. Acad. Sci. USA 116, 13249-13254 (2019).

30. Frachet, $M$. et al. Hidden magnetism at the pseudogap critical point of a cuprate superconductor. Nat. Phys. 16, 1064-1068 (2020).

31. Agterberg, D. F. et al. The physics of pair-density waves: cuprate superconductors and beyond. Annu. Rev. Condens. Matter Phys. 11, 231-270 (2020).

32. Miao, $\mathrm{H}$. et al. High-temperature charge density wave correlations in $\mathrm{La}_{1.875} \mathrm{Ba}_{0.125^{-}}$ $\mathrm{CuO}_{4}$ without spin-charge locking. Proc. Natl. Acad. Sci. USA 114, 12430-12435 (2017).

33. Arpaia, R. et al. Dynamical charge density fluctuations pervading the phase diagram of a Cu-based high- $T_{c}$ superconductor. Science 365, 906-910 (2019).

34. Yoshida, T. et al. Systematic doping evolution of the underlying Fermi surface of $\mathrm{La}_{2-x} \mathrm{Sr}_{x} \mathrm{CuO}_{4}$. Phys. Rev. B 74, 224510 (2006).

35. Horio, M. et al. Three-dimensional Fermi surface of overdoped La-based cuprates. Phys. Rev. Lett. 121, 077004 (2018).

36. Wen, J.-J. et al. Observation of two types of charge-density-wave orders in superconducting $\mathrm{La}_{2-x} \mathrm{Sr}_{x} \mathrm{CuO}_{4}$. Nat. Commun. 10, 3269 (2019).

37. Thampy, V. et al. Comparison of charge modulations in $\mathrm{La}_{1.875} \mathrm{Ba}_{0.125} \mathrm{CuO}_{4}$ and $\mathrm{YBa}_{2} \mathrm{Cu}_{3} \mathrm{O}_{6.6}$. Phys. Rev. B 88, 024505 (2013).

38. Lin, J. Q. et al. Strongly correlated charge density wave in $\mathrm{La}_{2-x} \mathrm{Sr}_{x} \mathrm{CuO}_{4}$ evidenced by doping-dependent phonon anomaly. Phys. Rev. Lett. 124, 207005 (2020).

39. Miao, $\mathrm{H}$. et al. Incommensurate phonon anomaly and the nature of charge density waves in cuprates. Phys. Rev. X 8, 011008 (2018).

40. Miao, H. et al. Formation of incommensurate charge density waves in cuprates. Phys. Rev. X 9, 031042 (2019).

41. Corboz, P., Rice, T. M. \& Troyer, M. Competing states in the $t-J$ model: uniform $d-$ wave state versus stripe state. Phys. Rev. Lett. 113, 046402 (2014).

42. Huang, E. W. et al. Numerical evidence of fluctuating stripes in the normal state of high- $T_{c}$ cuprate superconductors. Science 358, 1161-1164 (2017)

43. Zheng, B.-X. et al. Stripe order in the underdoped region of the two-dimensional Hubbard model. Science 358, 1155-1160 (2017).

44. Lorenzana, J. \& Seibold, G. Metallic mean-field stripes, incommensurability, and chemical potential in cuprates. Phys. Rev. Lett. 89, 136401 (2002).

45. Chaix, L. et al. Dispersive charge density wave excitations in $\mathrm{Bi}_{2} \mathrm{Sr}_{2} \mathrm{CaCu}_{2} \mathrm{O}_{8+\delta}$. Nat. Phys. 13, 952 (2017).

46. $\mathrm{Yu}, \mathrm{B}$. et al. Unusual dynamic charge correlations in simple-tetragonal $\mathrm{HgBa}_{2} \mathrm{CuO}_{4+\delta}$. Phys. Rev. X 10, 021059 (2020).

47. Zachar, O., Kivelson, S. A. \& Emery, V. J. Landau theory of stripe phases in cuprates and nickelates. Phys. Rev. B 57, 1422-1426 (1998).

48. Hücker, M. et al. Dzyaloshinsky-Moriya spin canting in the low-temperature tetragonal phase of $\mathrm{La}_{2-x-y} \mathrm{Eu}_{y} \mathrm{Sr}_{x} \mathrm{CuO}_{4}$. Phys. Rev. B 70, 214515 (2004).
49. Chen, X. et al. Charge density wave memory in a cuprate superconductor. Nat. Commun. 10, 1435 (2019).

50. Lee, P. A. Amperean pairing and the pseudogap phase of cuprate superconductors. Phys. Rev. X 4, 031017 (2014).

51. Berg, E. et al. Dynamical layer decoupling in a stripe-ordered high- $T_{c}$ superconductor. Phys. Rev. Lett. 99, 127003 (2007).

52. Yamada, K. et al. Doping dependence of the spatially modulated dynamical spin correlations and the superconducting-transition temperature in $\mathrm{La}_{2-x} \mathrm{Sr}_{x} \mathrm{CuO}_{4}$. Phys. Rev. B 57, 6165-6172 (1998).

\section{ACKNOWLEDGEMENTS}

We thank N. Christensen, G. Kotliar, J. Q. Lin, V. Thampy, A. Tsvelik, and W. G. Yin for insightful discussions, and J. Jiang and S. S. Zhang for technical support. This material is based upon work supported by the US Department of Energy (DOE), Office of Basic Energy Sciences. Work at Brookhaven National Laboratory was supported by the US Department of Energy, Office of Basic Energy Sciences, under contract no. DESC0012704. X-ray and photoemission measurements used resources at the 4-ID and 21-ID-1 beamlines of the National Synchrotron Light Source II, a US Department of Energy Office of Science User Facility operated for the DOE Office of Science by Brookhaven National Laboratory under contract no. DE-SC0012704. Additional X-ray measurements used resources at 4-ID-D in the Advanced Photon Source, a US Department of Energy (DOE) Office of Science User Facility operated for the DOE Office of Science by Argonne National Laboratory under contract no. DE-ACO2$06 \mathrm{CH} 11357$.

\section{AUTHOR CONTRIBUTIONS}

H.M., T.Y., K.K., E.V., and P.D.J. performed the ARPES measurements. H.M., G.F., R.J.K., D.G.M., C.S.N., R. A.-E., T.A., I.K.R., E.S.B., and M.P.M.D. performed the X-ray measurements. Y.L., G.D.G., M.O., K.K., and N.M. grew the LSCO samples and characterized their transport properties. H.M., P.D.J., and M.P.M.D. analyzed the data. H.M., J.M.T., and M.P.M.D. wrote the paper.

\section{COMPETING INTERESTS}

The authors declare no competing interests.

\section{ADDITIONAL INFORMATION}

Supplementary information The online version contains supplementary material available at https://doi.org/10.1038/s41535-021-00327-4

Correspondence and requests for materials should be addressed to H.M. or M.P.M.D.

Reprints and permission information is available at http://www.nature.com/ reprints

Publisher's note Springer Nature remains neutral with regard to jurisdictional claims in published maps and institutional affiliations.

Open Access This article is licensed under a Creative Commons Attribution 4.0 International License, which permits use, sharing, adaptation, distribution and reproduction in any medium or format, as long as you give appropriate credit to the original author(s) and the source, provide a link to the Creative Commons license, and indicate if changes were made. The images or other third party material in this article are included in the article's Creative Commons license, unless indicated otherwise in a credit line to the material. If material is not included in the article's Creative Commons license and your intended use is not permitted by statutory regulation or exceeds the permitted use, you will need to obtain permission directly from the copyright holder. To view a copy of this license, visit http://creativecommons. org/licenses/by/4.0/.

(c) The Author(s) 2021 